\section{Wischtests zum Drogennachweis}

T. Arndt

Bioscientia Institut für Medizinische Diagnostik GmbH, Ingelheim, Deutschland

Synonym(e) Drogenwischtest

\section{Englischer Begriff Drugwipe}

Definition Drugwipe II sind Drogenschnellteste der Securetec AG, München, die zur Vor-Ort-Kontrolle von Drogenkonsum, z. B. im Straßenverkehr, eingesetzt werden.

Beschreibung Das Testfeld des Wischtests wird mehrfach über die Hautoberfläche (z. B. Achselhöhle) gewischt. Der Drogen- oder Drogenmetabolitnachweis erfolgt immunologisch mit spezifischen Antikörpern (s. a. > Teststreifen) innerhalb weniger Minuten. Als Alternativmaterial können
Speichel und Oberflächen auf Drogen geprüft werden. Derzeit stehen Systeme zum Nachweis der Amphetamine/ Methamphetamin (Ecstasy) (s. a. \ Amphetamine), > Benzodiazepine, $>$ Cannabinoide, $\triangleright$ Kokain und Opiate zur Verfügung. Die Nachweisgrenze liegt zwischen $5-200 \mathrm{ng} / \mathrm{mL}$ (Speichel, Schweiß) bzw. 2-20 ng/ $\mathrm{cm}^{2}$ (Oberflächen).

Ein positiver Wischtest- oder sonstiger Drogenschnelltestbefund muss unter forensischen Bedingungen generell durch weiterführende Untersuchungen, z. B. zur Beurteilung einer aktuellen Drogenbeeinflussung durch Blutanalyse, mit physikochemischen Analyseverfahren, zumeist $>$ Massenspektrometrie, vertieft und bestätigt werden (sog. \ Bestätigungsuntersuchungen). Ein Schnelltest dient als eine erste Entscheidungshilfe, z. B. bei polizeilichen Maßnahmen, wobei ein Schnelltestbefund vor Gericht nicht beweiskräftig ist.

\section{Literatur}

www.securetec.net 